OPEN ACCESS

Edited by:

James Cheng-Chung Wei,

Chung Shan Medical University Hospital, Taiwan

Reviewed by:

HuangHsi Chen,

Chung Shan Medical University Hospital, Taiwan

Qibing Xie,

Sichuan University, China

${ }^{*}$ Correspondence:

Guoyong Ding

dgy-153@163.com

${ }^{\dagger}$ These authors have contributed equally to this work and share first authorship

Specialty section: This article was submitted to Autoimmune and Autoinflammatory Disorders, a section of the journal

Frontiers in Immunology

Received: 01 September 2021 Accepted: 11 October 2021 Published: 22 October 2021

Citation:

Zhang $X$, Sun Z, Zhou A, Tao L, Chen $Y$, Shi X, Yin J, Sun Z and Ding G (2021) Association Between Infections and Risk of Ankylosing Spondylitis: A Systematic Review and Meta-Analysis.

Front. Immunol. 12:768741.

doi: 10.3389/fimmu.2021.768741

\section{Association Between Infections and Risk of Ankylosing Spondylitis: A Systematic Review and Meta-Analysis}

\author{
Xiao Zhang ${ }^{1,2,3 \dagger}$, Zhe Sun ${ }^{4 \dagger}$, Aihong Zhou ${ }^{4}$, Lei Tao ${ }^{4}$, Yingxin Chen ${ }^{1,2}$, Xinyu Shi ${ }^{1,2}$, \\ Jia Yin ${ }^{3}$, Zheng Sun ${ }^{2}$ and Guoyong Ding ${ }^{3,4 *}$ \\ ${ }^{1}$ School of Nursing, Shandong First Medical University \& Shandong Academy of Medical Sciences, Taian, China, 2 School of \\ Clinical Medline, Shandong First Medical University \& Shandong Academy of Medical Sciences, Jinan, China, ${ }^{3}$ School of \\ Public Health, Shandong First Medical University \& Shandong Academy of Medical Sciences, Taian, China, ${ }^{4}$ The Second \\ Affiliated Hospital of Shandong First Medical University, Taian, China
}

Background: Previous literature on the association between infections and the risk of developing ankylosing spondylitis (AS) presented controversial results. This meta-analysis aimed to quantitatively investigate the effect of infections on the risk of AS.

Methods: We searched the PubMed, Embase, and Web of Science databases until March 26, 2021 for analytical epidemiological studies on the association between infections and the risk of AS. Fixed or random effect models were used to calculate total risk estimates based on study heterogeneity. Subgroup analysis, and sensitivity analysis were also performed. Publication bias was estimated using funnel plots and Begg's test.

Results: Six case-control articles $(n=1,296,239)$ and seven cohort articles $(n=7,618,524)$ were incorporated into our meta-analysis. The pooled odds ratio (OR) from these casecontrol studies showed that infections were associated with an increased risk of AS (OR=1.46, 95\% confidence interval [Cl], 1.23-1.73), and the pooled relative risk (RR) from the cohort studies showed the same findings $(R R=1.35,95 \% \mathrm{Cl}, 1.12-1.63)$. Subgroup analysis showed that infections in participants with unadjusted comorbidities ( $\mathrm{OR}=1.66$, $95 \% \mathrm{Cl}, 1.35-2.03)$, other types of infection ( $\mathrm{OR}=1.40$, 95\% Cl, 1.15-1.70), and infection of the immune system $(\mathrm{OR}=1.46,95 \% \mathrm{Cl}, 1.42-1.49)$ were associated with the risk of $\mathrm{AS}$ in case-control studies. In cohort studies, infections with adjusted comorbidities $(R R=1.39,95 \% \mathrm{Cl}, 1.15-1.68)$, viral infection $(R R=1.43,95 \% \mathrm{Cl}, 1.22-1.66)$, other types of infection $(R R=1.44,95 \% \mathrm{Cl}, 1.12-1.86)$, and other sites of infection ( $R R=1.36$, $95 \% \mathrm{Cl}, 1.11-1.67)$ were associated with an increased risk of AS.

Conclusions: The findings of this meta-analysis confirm that infections significantly increase the risks of AS. This is helpful in providing an essential basis for the prevention of AS via the avoidance of infections.

Keywords: ankylosing spondylitis, infections, analytical epidemiology, systematic review, meta-analysis 


\section{INTRODUCTION}

Ankylosing spondylitis (AS), a complex autoimmune inflammatory rheumatic disease, has long been considered the archetype of spondyloarthritis (SpA). Common symptoms of AS include arthritic symptoms (such as inflammatory back pain, muscle spasms, and sacroiliac arthritis), potential extra-articular symptoms (such as uveitis, psoriasis, and inflammatory bowel syndrome), and the involvement of the heart, bone, lung, kidneys, and skin $(1,2)$. The worldwide prevalence of AS ranges between $0.07 \%$ and $0.32 \%$ (3). In addition, clinical symptoms of patients with AS usually appear between the ages of 26 and 45 years. Men also are more likely to suffer from AS than women, the prevalence being two to three times higher in men than in women (4-6).

The pathogenesis of AS is complex and multifactorial. Early studies have confirmed that AS is strongly associated with the inheritance of HLA allele B27, which might misfold in the endoplasmic reticulum (ER), leading to the upregulation of interleukin (IL)-23 in dendritic cells (7-9). It may also result in the presentation of intracellular peptides to $\mathrm{T}$ cells, which may trigger cross reactions, leading to tissue inflammation $(10,11)$. Several recent studies have emphasized the critical role of intestinal flora dysregulation in the development and progression of AS, and have suggested that $60 \%$ of AS patients are associated with subclinical intestinal inflammation (12-14). This might be related to the imbalance of IL-17 or IL-23 cytokines caused by the activation a Th17-mediated immune response by intestinal dysbiosis (15). In contrast, the role of environmental factors in the etiology of AS is far from clear. One of the most popular theories presume that the onset of AS in susceptible individuals may be caused by infections (16), and that infections have the potential to modulate and attenuate immune responses.

The underlying pathogenic mechanisms for linking infections and AS involve changes in target cells and immune cells, and antigenic cross-reactions between microbial and host determinants (17). Infections might cause the quantitative reduction in specific $\mathrm{T}$ cells and the host defense defect against the infections that allows microbial antigens to reach the joint (18). The association between the infections and AS may be via IL-17 or C reactive protein levels that can induce inflammatory response $(10,19)$. In addition, certain microbial infections may reduce $\mathrm{CD} 4+\mathrm{T}$ cells, and protein fragments released by dying CD4 lymphocytes may induce autoreactive CD8 lymphocytes (20). There is evidence of significantly elevated levels of IL-6 and TNF-a in AS patients, which might be caused by infections (21).

Numerous studies have investigated AS-related infections, including bacterial $(10,17,18,22)$, viral $(17,19,20,23)$, fungal (11), and those by microorganisms with sizes between those of bacteria and viruses $(18,24,25)$. The infected sites include the respiratory $(18,24-26)$, immune $(20,23,26,27)$, digestive (10, $22,26,28)$, and genitourinary systems (19). However, there is no consensus on the association between infections and the risk of AS. To our knowledge, no systematic review and meta-analysis to date has investigated the effect of infections on the risk of AS. Therefore, in order to obtain a more convincing conclusion, this study aimed to review all the relevant studies and summarise the findings, in order to investigate the association between infections and AS.

\section{MATERIALS AND METHODS}

The current study was developed according to the guidelines for the Meta-Analysis of Observational Studies in Epidemiology (MOOSE) (29) and Preferred Reporting Items for Systematic Review and Meta-Analyses (PRISMA, Supplementary Table 1) (30). The protocol is presented in Supplementary Data.

\section{Search Strategy}

We systematically searched the PubMed, Embase, and Web of Science electronic databases to identify such literature published up until March 26, 2021, using terms related to infection and AS. The search strategy was developed and implemented under the guidance of experts on library services from Shandong First Medical University. The main search strategy involved the following: (spondylitis, ankylosing OR spondyloarthritis ankylopoietica OR ankylosing spondylarthritis OR ankylosing spondylarthritides OR spondylarthritides, ankylosing) AND (infections OR enteritis OR salmonella OR pneumonia OR klebsiella pneumoniae OR urogenital infections OR paradentitis OR tonsillitis OR infection of the upper respiratory tract $\mathrm{OR}$ appendicitis $\mathrm{OR}$ gastritis $\mathrm{OR}$ helicobactor pylori OR virus) AND (case-control study OR retrospective study OR cohort study OR prospective study OR longitudinal study OR follow-up study). The complete search strategy of the three databases is listed in Supplementary Table 2. Moreover, only English- or Chinese-language literature was retrieved from the databases as the investigators were proficient in both these languages. The lists of references from all of the included studies were manually checked to identify possible additional studies.

\section{Selection Criteria}

Studies were included according to the following criteria: (1) the study design was a cohort or case-control study; (2) the studies defined infections using self-reporting, clinical diagnosis, or basic medical experiment, and focused on infections that developed before AS did; (3) the outcome of interest was AS; and (4) the studies provided the effect size (relative risk [RR], hazard ratio [HR], or odds ratio [OR] with $95 \%$ confidence interval [CI]) or raw data that could be used to calculate RR, HR, or OR. The exclusion criteria were as follows: (1) non-human-based studies; (2) studies that were poster presentations, reviews, conference summaries, or dissertations; and (3) the scores of quality evaluation according to the Newcastle-Ottawa Scale (NOS) were $<4$ (31). In the situation of multiple eligible studies from the same population, only the study with the largest number of individuals was included. Two authors (X.Z. and A.Z.) independently screened titles and abstracts initially and then evaluated full-text articles to ensure the included studies met the eligible inclusion criteria. Any disagreement between them was settled by another author (G.D.). 


\section{Data Extraction and Quality Assessment}

According to the study design, the included studies were divided into two extraction forms of case-control studies and cohort studies. The following data were extracted from the eligible casecontrol studies using a customized form: the first name of the first author, year of publication, study location, types of infection, definition of infection, definition of AS, age, sex, sample size, adjustment for potential confounding factors, and estimates of association. The follow-up duration in cohort studies was also included. The Cochrane Non-randomized Studies Methods Working Group recommended the use of the NOS to assess the quality of observational studies (range: 0-9 stars) (32). According to the score stars of the NOS, the included studies were defined as low- (1-3 stars), moderate- (4-6 stars), and highquality (7-9 stars). Therefore, if the study obtained $\geq 4$ stars, it was considered to have an above-moderate quality and, thus, was incorporated into our meta-analysis (31). Data extraction and quality assessment were conducted by two independent investigators (L.T. and Y.C.), and disagreements between them were resolved through negotiation with a third researcher (Z.S.).

\section{Statistical Analysis}

The statistical analyses were performed using Stata 15.1 software (Stata Corp, College Station, TX, USA). All of the tests were bilateral, and $P$ values $<0.05$ were considered statistically significant, though $P$ values $>0.1$ illustrated no heterogeneity among studies in the heterogeneity test (33). ORs, RRs, or HRs and their corresponding 95\% CIs were considered to be the effect values of different infections on the risk of AS. The pooled OR and RR with their corresponding 95\% CIs were used in casecontrol and cohort studies, respectively, to assess the association of infection with the risk of AS. We used the $Q$ test and the $I^{2}$ statistic to detect heterogeneity among the studies. $I^{2}$ describes the percentage of total variation due to heterogeneity among studies rather than due to chance (34). In the presence of high heterogeneity $\left(I^{2}>50 \%\right)$, the Dersimonian and Laird random effects model (REM) was adopted as the pooling method; otherwise, the Mantel-Haensze fixed effects model (FEM) was applied as the pooling method.

Subgroup analyses were performed based on adjusting for comorbidities, infection type, and infection site. In addition, considering that publication year, study location, sample size, definition of infection, and duration of follow-up (only in cohort studies) may affect between-study heterogeneity, subgroup analysis was also conducted based on these possible factors. Sensitivity analyses were performed to validate the stability of pooled ORs of case-control literature and pooled RRs of cohort literature by removing each individual study. In addition, we used the funnel plot and Begg's test to assess publication bias.

\section{RESULTS}

\section{Literature Search and Study Selection}

The flowchart of the literature search and study selection process is represented in Figure 1. Using three electronic databases and running the search strategy, a total of 4,584 potentially relevant articles were identified. In total, 1,358 duplicate articles were excluded. An additional 3,226 articles were excluded by screening for the title and abstract, leaving 24 articles for the full-text review. Screening via hand-searching found 1 relevant article. An additional 12 articles were excluded because they did not meet the inclusion criteria. Therefore, 13 articles that met the inclusion criteria were ultimately included $(10,11,17-20$, 22-28).

\section{Study Characteristics}

There were six case-control design articles $(18,22,25-28)$ and seven cohort study design articles $(10,11,17,19,20,23,24)$. It must be noted that one article involved two case-control studies (28). Therefore, we included 13 articles with 14 studies. The characteristics of the included studies, which were published from 2004 to 2020, are summarized in Supplementary Tables 3-6. The case-control studies included 1,296,239 participants; the cohort studies, 7,618,524 participants.

Seven studies were from Asia $(11,19,22-25,27)$, five, from Europe $(10,17,26,28)$, and two, from North America $(18,20)$. The mean NOS star score of the methodological quality of the studies was 7.9. Two articles (with three studies) obtained 5 stars $(25,28)$; two studies, 7 stars $(17,23)$; two studies, 8 stars $(10,26)$; and seven studies, 9 stars $(11,18-20,22,24,27)$. The follow-up duration ranged from 3.6 to 34 years in the cohort studies. All studies involved both men and women $(10,11,18-20,22-28)$ except the study by Nielsen et al. (17). Nine studies defined the infection using clinical diagnosis $(11,17,19,20,22-24,26,27)$, two studies (in the same article) defined the infection using selfreport (28), and three studies defined the infection using laboratory tests in etiologic diagnoses $(10,18,25)$. Thirteen studies reported one type of infection $(10,11,19,20,22-28)$, and one study reported four types of infections (18). In the studies, except for those by Feng et al. and Yen et al., the risk estimates were adjusted for confounding variables, such as age, sex, urbanization, income, comorbidities, body mass index, educational attainment, smoking status, and alcohol consumption $(10,11,17-20,22,24,26-28)$.

\section{Infection and the Risk of AS}

Figures 2, 3 show the association between infections and the risk of AS in seven case-control studies $(18,22,25-28)$ and seven cohort studies $(10,11,17,19,20,23,24)$, respectively. There were significant heterogeneities among the seven case-control studies $\left(P_{Q}<0.001 . I^{2}=88.9 \%\right)$, and the seven cohort studies $\left(P_{Q}<0.001\right.$. $\left.I^{2}=70.5 \%\right)$. Therefore, REM was used to pool the OR for casecontrol studies and RR for cohort studies. Our meta-analysis showed that, compared to the control group, the infection group was significantly associated with an increased risk of AS. The pooled OR calculated by REM was 1.46 (95\% CI, 1.23-1.73) for the case-control studies, and the pooled RR was 1.35 (95\% CI, 1.12-1.63) for the cohort studies.

\section{Subgroup Analysis}

Table 1 shows the subgroup analysis based on comorbidities (adjusted vs. unadjusted), infection type (bacterial vs. viral $v s$. other), and infection site (immune system $v s$. other). 


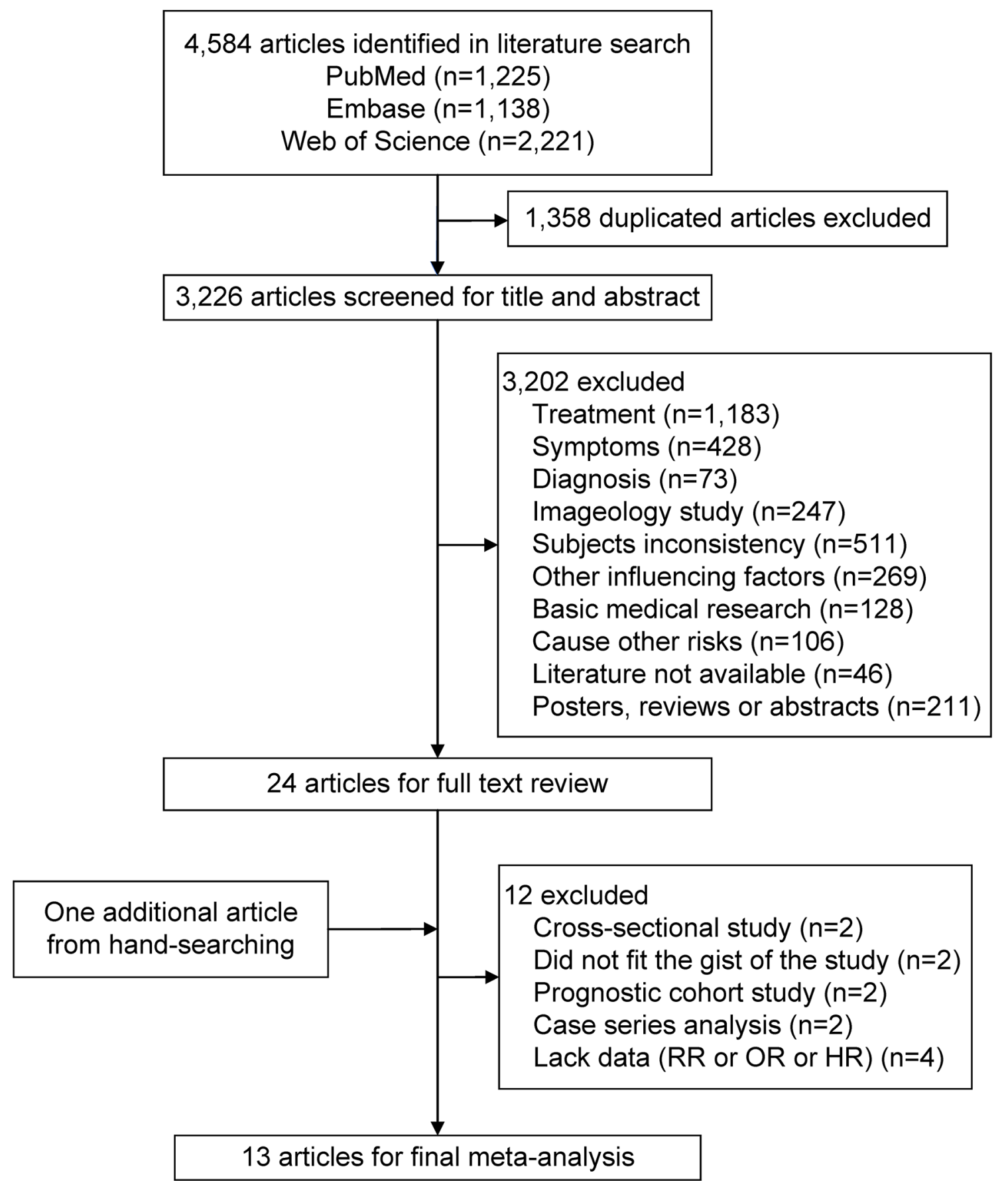

FIGURE 1 | Flowchart of the selection of studies included in this meta-analysis. HR, hazard ratio; OR, odds ratio; RR, relative risk.

In the case-control studies, our analysis showed no significant difference between adjusted comorbidities ( $\mathrm{OR}=1.26,95 \% \mathrm{CI}$, 0.94-1.70) and unadjusted comorbidities (OR=1.66, 95\% CI, $1.35-2.03)(P=0.139)$. Similar results were reported with the infection type (bacterial, $\mathrm{OR}=1.31,95 \% \mathrm{CI}, 0.61-2.78$; other, $\mathrm{OR}=1.40,95 \% \mathrm{CI}, 1.15-1.70 ; P=0.864)$ and the infection site (immune system, $\mathrm{OR}=1.46,95 \% \mathrm{CI}, 1.42-1.49$; other, $\mathrm{OR}=1.27$, 95\% CI, 0.96-1.69; $P=0.347)$.

In the cohort studies, our analysis showed no statistically significant difference between adjusted comorbidities $(R R=1.39$, 95\% CI, 1.15-1.68) and unadjusted comorbidities ( $R R=0.86,95 \%$ CI, $0.45-1.63 ; P=0.157)$. No differences were also found in the 


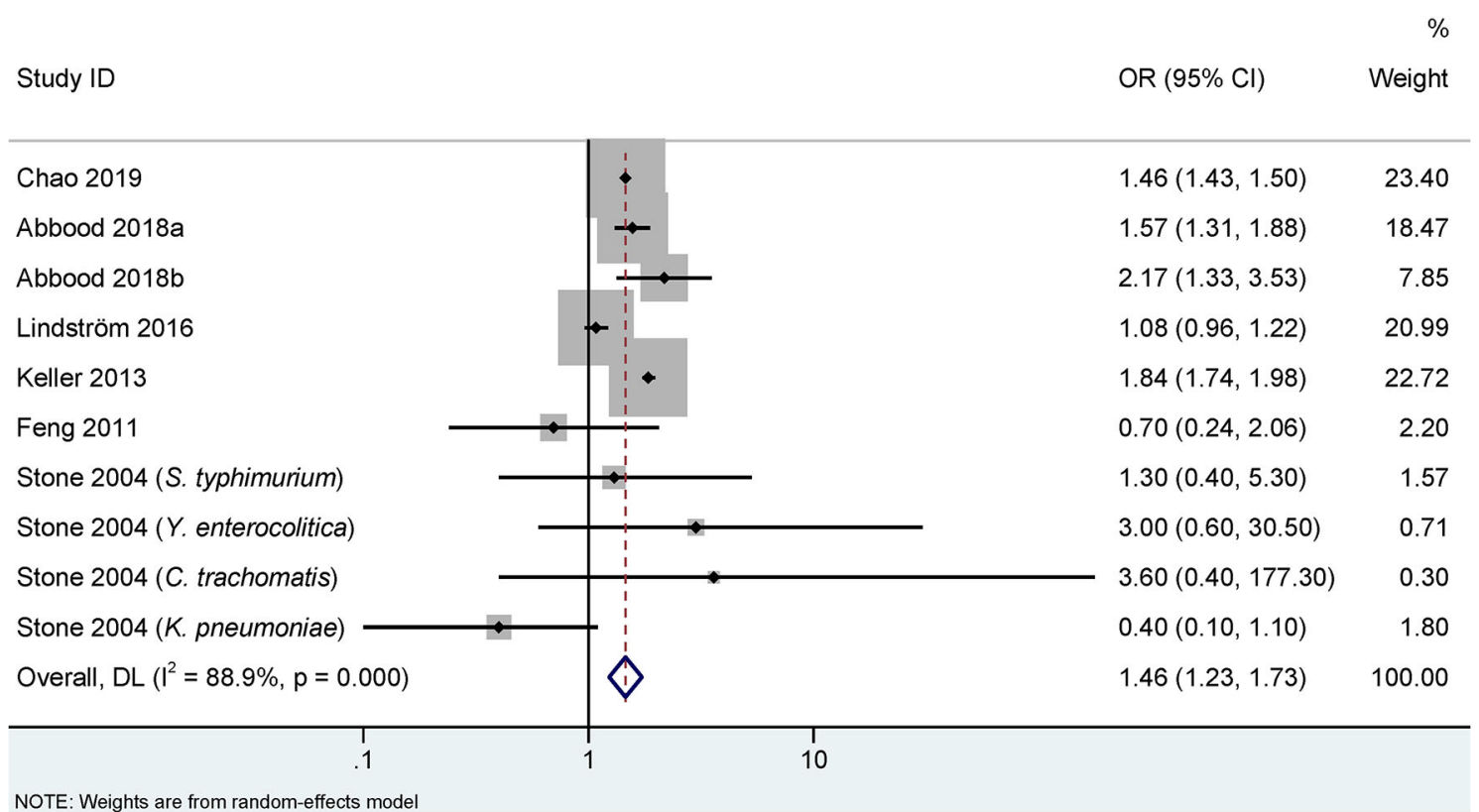

FIGURE 2 | Random effects meta-analysis of the association between infections and ankylosing spondylitis in case-control studies. Cl, confidence interval; OR, odds ratio. a represents case-control study of self-reported ankylosing spondylitis; b represents case-control study of clinical recorded ankylosing spondylitis.

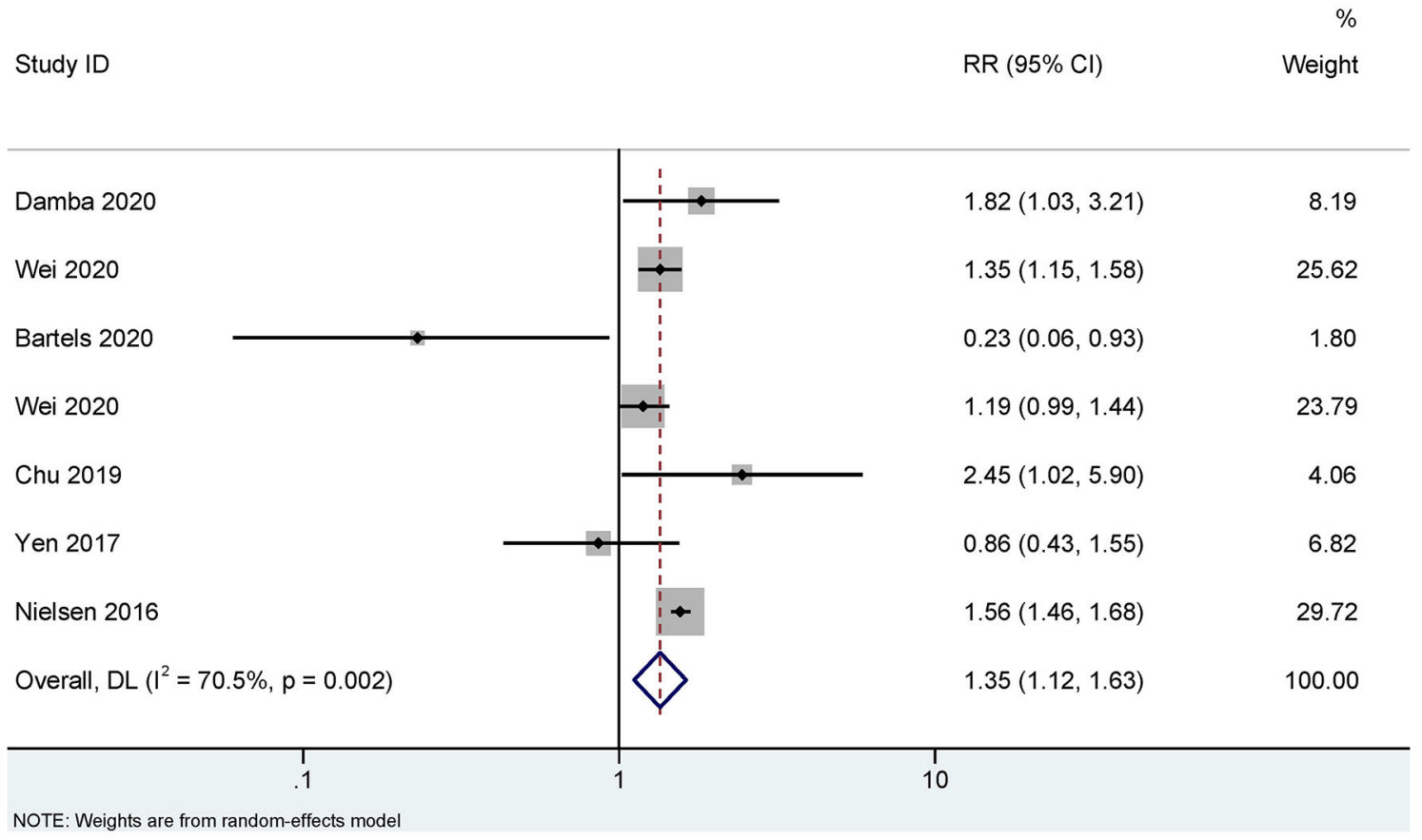

FIGURE 3 | Random effects meta-analysis of the association between infections and ankylosing spondylitis in cohort studies. Cl, confidence interval; RR, relative risk. 
TABLE 1 | The subgroup analyses of the association between infections and the risk of AS.

\begin{tabular}{|c|c|c|c|c|}
\hline Subgroups & No. of items & OR/RR/HR (95\% Cl) & $I^{2}(\%)$ & Chi-square test $P$-value \\
\hline Comorbidities & & & & 0.139 \\
\hline Adjusted & 2 & $1.26(0.94-1.70)$ & 95.7 & \\
\hline Infection Type & & & & 0.864 \\
\hline Bacterial & 4 & $1.31(0.61-2.78)$ & 55.3 & \\
\hline Other ${ }^{*}$ & 6 & $1.40(1.15-1.70)$ & 82.7 & \\
\hline Other** & 10 & $1.27(0.96-1.69)$ & 86.7 & \\
\hline \multicolumn{5}{|l|}{ Cohort studies } \\
\hline Comorbidities & & & & 0.157 \\
\hline Adjusted & 6 & $1.39(1.15-1.68)$ & 71.6 & \\
\hline Unadjusted & 1 & $0.86(0.45-1.63)$ & 0.0 & \\
\hline Infection Type & & & & 0.766 \\
\hline Immune system & 2 & $1.27(0.61-2.65)$ & 66.0 & \\
\hline Other** & 5 & $1.36(1.11-1.67)$ & 76.6 & \\
\hline
\end{tabular}

$A S$, ankylosing spondylitis; $\mathrm{Cl}$, confidence interval; $H R$, hazard ratio; $O R$, odds ratio, $R R$, relative risk.

*Other types of infection include fungi, chlamydia, and mycoplasma.

${ }^{*}$ Other sites of infection include the digestive system, respiratory system, and genitourinary system.

infection type (bacterial, $\mathrm{RR}=0.70,95 \% \mathrm{CI}, 0.10-4.78$; viral, $\mathrm{RR}=1.43,95 \% \mathrm{CI}, 1.22-1.66$; other, $\mathrm{RR}=1.44$, 95\% CI, $1.12-$ 1.86; $P=0.766$ ) and infection site (immune system, $\mathrm{RR}=1.27,95 \%$ CI, 0.61-2.65; other, $\mathrm{RR}=1.36,95 \% \mathrm{CI}, 1.11-1.67 ; P=0.863)$.

We also conducted subgroup analysis based on publication year, study location, sample size, definition of infection, and duration of follow-up. As shown in Supplementary Table 7, the results showed no significant difference between those subgroups in the case-control studies, which indicated that these above factors were not the source of heterogeneity in case-control studies. In the cohort studies, only for definition of infection, there was a significant deference between clinical diagnosis $\left(\mathrm{RR}=1.39,95 \% \mathrm{CI}, 1.19-1.63, I^{2}=62.3 \%\right)$ and basic medical experiment $\left(\mathrm{RR}=0.23,95 \% \mathrm{CI}, 0.06-0.91, I^{2}=0 \%, P=0.010\right)$. Definition of infection might be one of the sources of heterogeneity in the cohort studies.

\section{Sensitivity Analysis and Publication Bias Detection}

As shown in Figure 4, the results of sensitivity analysis showed that no individual study significantly influenced the pooled OR in the case-control studies. However, the study by Nielsen et al.
A

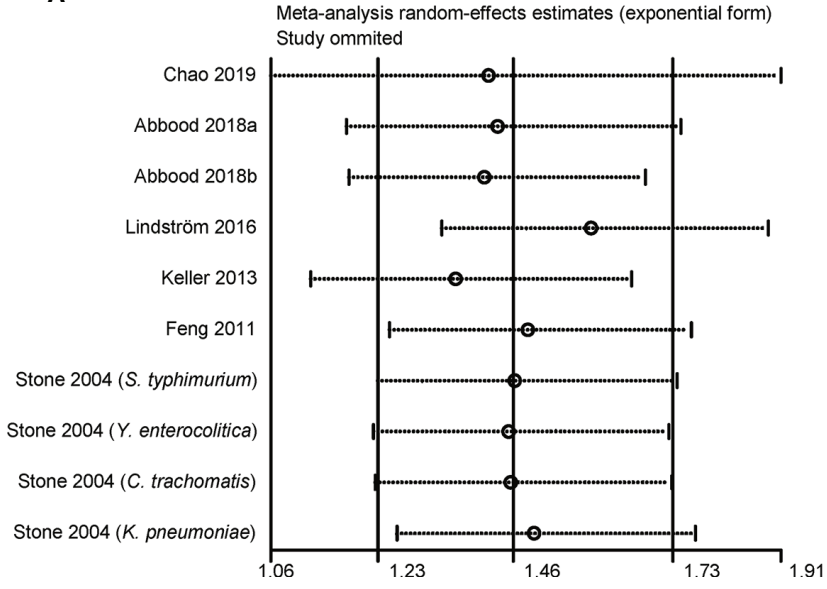

B

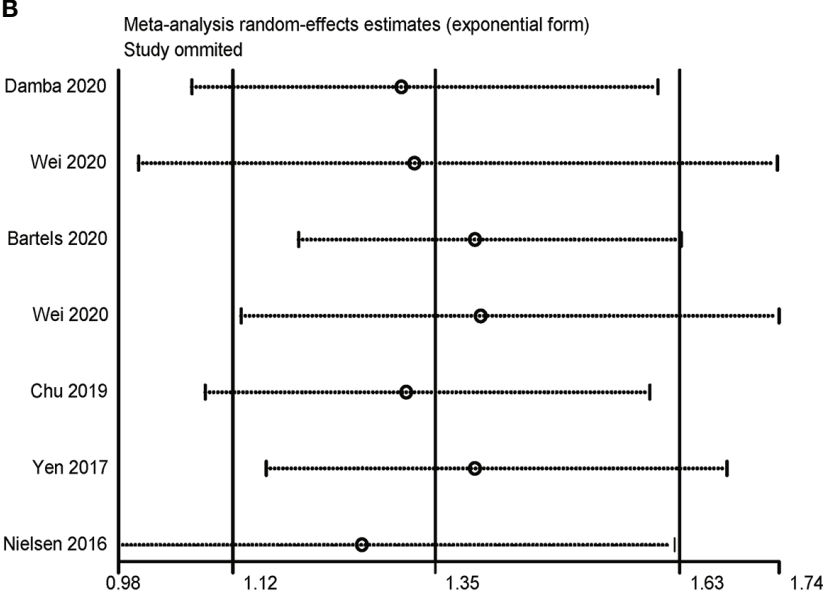

FIGURE 4 | Sensitivity analysis of the association between infections and ankylosing spondylitis. (A) Case-control study; a represents case-control study of selfreported ankylosing spondylitis; b represents case-control study of clinical recorded ankylosing spondylitis; (B) Cohort study. 
affected the pooled RR in the cohort studies. The pooled RR was 1.26 (95\% CI, $\left.0.98-1.62 ; I^{2}=58.6 \%\right)$ by omitting this study.

The funnel plots for estimating publication bias were roughly symmetrical for the case-control (Figure 5A) and cohort studies (Figure 5B). No publication bias was detected by Begg's test for the case-control $(P=0.721)$ and cohort studies $(P=0.368)$.

\section{DISCUSSION}

To the best of our knowledge, this meta-analysis is the first study to investigate the association between infections and the risk of developing AS. The current meta-analysis included seven casecontrol studies with 1,296,239 participants and seven cohort studies with 7,618,524 participants. The results of this study confirm that infections play an important role in the risk of AS. Determining the effect of infections on AS would be beneficial for the identification of those who are at higher risk of AS as reasonable preventive intervention can be conducted for this population, enabling a farreaching significance for the prevention of AS.

The results showed that infections are associated with an increased risk of AS in both the case-control and cohort studies. Our findings are consistent with most of the studies, including the four studies from Asia $(19,22,24,27)$, three studies from Europe $(17,28)$, and one study from North America (20). However, the study by Bartels et al. showed opposite results (10), in that previous Helicobacter pylori (H.pylori) infection may reduce the risk for developing AS. Another study found that H. pylori infection was eradicated in more than $80 \%$ of cases in the same cohort as that of Bartels et al. (35), indicating that $H$. pylori leaves a protective potential for the development of AS later in life when it is eradicated (10). Furthermore, the microbiota in the gastrointestinal tract changes after the eradication of $H$. pylori, which may have an impact on AS development $(36,37)$.

Our subgroup analyses showed that there was an association between infection and the risk of AS after adjusting for comorbidities in the cohort studies, which suggested that the comorbidities are a remarkably important confounding factor in cohort studies, and that we must control and adjust it. However, an association between infection and the risk of AS was found in the case-control studies without adjusted comorbidities. This is due to the nature of the case-control study design. As one of the matching factors of case-control studies, comorbidities may be matched in the design stage, cancelling the need to adjust for comorbidities in the statistical analysis stage.

With regards to the infection types, we did not observe that bacterial infections contribute to the risk of AS. In case-control studies, only the study by Keller et al. showed that there is an association between AS and a prior diagnosis of chronic periodontitis, which is characterized by an oral bacterial infection (22). This may be because rheumatic diseases and chronic periodontitis share pathogenic factors, including a dysfunction of inflammatory mechanisms and an imbalance of proinflammatory and anti-inflammatory cytokines (22, 38-40). In the cohort studies, the study by Bartels et al. showed that $H$. pylori may be a protective factor for AS (10). The study of Nielsen et al. also showed that bacterial infection is associated with the development of AS in the general population (17). More cohort studies are needed to verify whether bacterial infection causes AS. The result of the subgroup of other infection types was that other infection types are associated with AS in both the case-control and cohort studies. In our analysis, other types of infection included fungal, chlamydia, and mycoplasma. The pathogenesis of AS due to other types of infection is far from clear. For example, one study suggested that AS could be induced after exposure to Candida albicans through a $\mathrm{T}$ cell-driven model towards Th17 responses (11). Another study suggested that Mycoplasma pneumoniae has a significant impact on immune cells and the immune system of the host, including polyclonal activation of $\mathrm{T}$ and $\mathrm{B}$ cells and the secretion of related cytokines (24), leading to a breakdown of immune-tolerance. In addition, subgroup analysis indicated that viruses play an important role in the risk of AS in the cohort studies. One study suggests that viruses (e.g., human
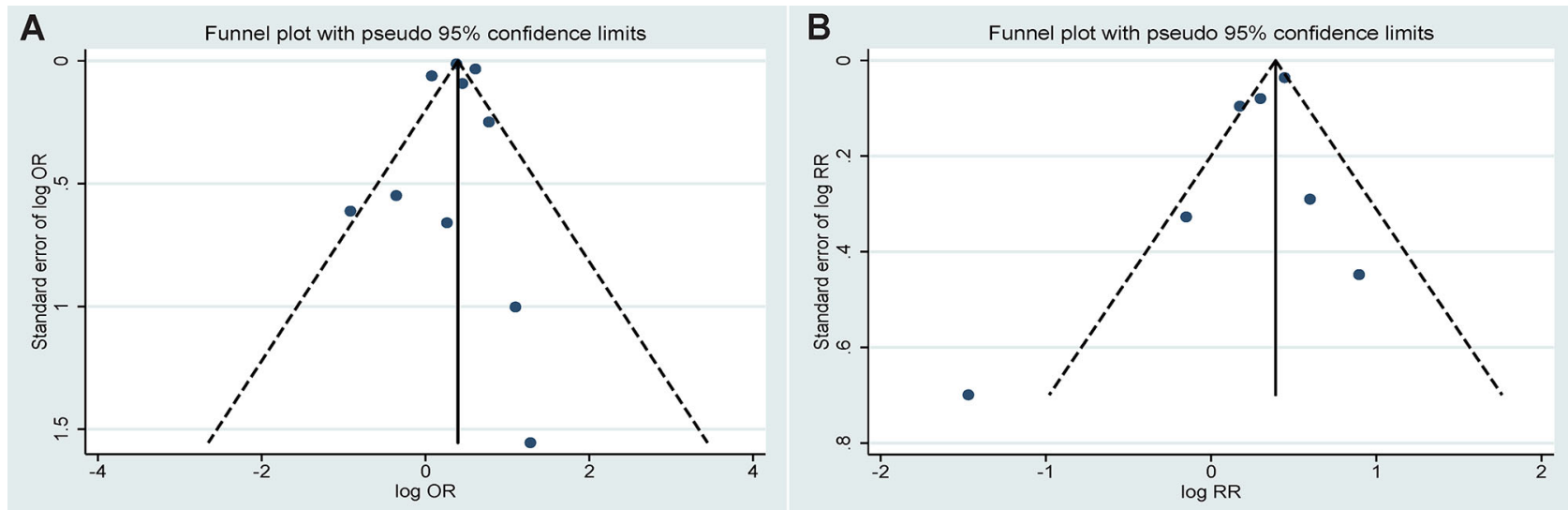

FIGURE 5 | Funnel plots corresponding to the random effects meta-analysis of the association between infections and ankylosing spondylitis. (A) Case-control study ( $P=0.721$ by Begg's test); (B) Cohort study ( $P=0.368$ by Begg's test). 
papillomavirus) might lead to inflammatory or immune-mediated disease by activating the pathogenic IL-23/IL-17 axis, resulting in elevated serum levels of Th17 cells, IL-17, and IL-23, and the imbalance of IL-17A/IL-23 cytokines (19). In the subgroup analysis of infection sites, we found that the infection of the immune system was significantly associated with the risk of AS in the case-control studies. Some immune organs, such as the tonsils, are involved in allergens tolerance by generating allergen-specific $\mathrm{FOXP}^{+}$regulatory $\mathrm{T}$ cells, suggesting that they are critical in the development of immune-tolerance (41). Some studies postulated that the alteration of immune tolerance in patients with tonsillitis might lead to the inflammatory disorders in autoimmune arthritis, including AS; therefore, tonsillitis might be aggravated by spondylitis, leading to the diagnosis of AS $(26,27)$. In addition, the higher risks of AS among infected people might be explained by HIV-induced antigen-driven immune responses (42), T cell imbalance (43), and molecular mimicry located between HIV protein and self-antigens (44). For the cohort studies, the infections in other sites were significantly associated with the risk of AS, which indicates that AS might be triggered by respiratory tract infections and genitourinary system infections. The pathogenesis of AS caused by the infection in the genitourinary system is mixed. In one of them, human papillomavirus in the genitourinary system might lead to AS by activating the IL-23/IL-17 axis (19). For the respiratory system, Klebsiella pneumoniae might lead to a decrease in the number of specific $\mathrm{T}$ cells, which could reflect an insufficient in the host's defense against Klebsiella, thereby allowing AS to be affected by bacterial antigens that reach the joint (18). In our study, we found that some design types were meaningful and some were not for the same subgroups, which might be related to the small number of included articles or the large heterogeneity between the included studies.

This meta-analysis has the following strengths: pooled effect values were analysed according to the different study design, and we grouped the studies according to the types and sites of infection to determine whether these factors were associated with the risk of AS. This study included analytical epidemiological studies to determine the risk of AS, and the sample size was large. The included studies were adjusted for potential confounding variables, which improved the accuracy of risk estimates.

However, some limitations have affected the current study. First, although heterogeneity was explored via subgroup analysis, it was still very high, which may be related to age, sex distribution of participants, definition of infection, diagnosis of AS, etc. The subgroup analysis suggested that definition of infection was one of sources of heterogeneity in the cohort studies, which indicated that the possible disagreement between measurement methods might be a source of misclassification. But heterogeneity within subgroups remained high. In addition, although we extracted the definition of AS, only two articles declared that the diagnosis of AS was based on the Amor criteria $(18,25)$. As most of articles were retrospective, the International Classification of Diseases codes for the diagnoses of AS were based on records made by physicians and hospitals rather than a prospective clinical setting; thus, we could not set uniform criteria for the definition of AS, which also may result in heterogeneity. Second, this meta-analysis included only English- and Chinese-language articles, and qualified articles in other languages were not included in the analysis, which might have affected the pooled estimated value. Third, our pooled effect is affected by the study by Nielsen et al. in the cohort studies. However, the study by Nielsen et al. has the largest weight when synthesizing RRs across studies, because of its large sample size, narrow confidence interval, and high quality. When the study by Nielsen et al. was omitted in the sensitivity analysis, the pooled effect estimate was affected by some lowquality studies due to the increased weights. Thus, more large cohort studies are recommended in the future to assess the impact of infections on the risk of AS.

In conclusion, this meta-analysis confirms that there is an association between infections and an increased risk of AS, although the included studies suffered from high heterogeneity. As much as the mechanism of infection and the effect of bacterial and viral infections on AS has not yet been determined, further studies, particularly more higher quality prospective cohort studies and case-control studies, are required to verify that there is a true cause-and-effect relationship between infections and the risk of developing AS.

\section{DATA AVAILABILITY STATEMENT}

The original contributions presented in the study are included in the article/Supplementary Material. Further inquiries can be directed to the corresponding author.

\section{AUTHOR CONTRIBUTIONS}

GD and XZ designed the study protocol. XZ, XS, and JY conducted the literature search. XZ, AZ, and GD retrieved and selected the articles. LT, YC, and ZhengS conducted data extraction. $\mathrm{XZ}$ and ZheS performed the statistical analysis of the data. XZ, ZheS, and GD wrote the manuscript draft. GD and ZhengS supervised the study. All authors contributed to the article and approved the submitted version.

\section{FUNDING}

This work was supported by the Natural Science Foundation of Shandong Province for the General Program (Grant No. ZR2020MH339). The funder had no role in study design, data collection and analysis, decision to publish, or preparation of the manuscript.

\section{SUPPLEMENTARY MATERIAL}

The Supplementary Material for this article can be found online at: https://www.frontiersin.org/articles/10.3389/fimmu.2021. 768741/full\#supplementary-material 


\section{REFERENCES}

1. Sieper J, Poddubnyy D. Axial Spondyloarthritis. Lancet (2017) 390:73-84. doi: 10.1016/s0140-6736(16)31591-4

2. Ajmani S, Keshri A, Srivastava R, Aggarwal A, Lawrence A. Hearing Loss in Ankylosing Spondylitis. Int J Rheum Dis (2019) 22:1202-8. doi: 10.1111/1756$185 x .13560$

3. Dean LE, Jones GT, MacDonald AG, Downham C, Sturrock RD, Macfarlane GJ. Global Prevalence of Ankylosing Spondylitis. Rheumatol (Oxford) (2014) 53:650-7. doi: 10.1093/rheumatology/ket387

4. Masi AT, Wilkins WR. Does Male:Female Sex Ratio in Ankylosing Spondylitis Change With Age? J Rheumatol (1996) 23:947-8.

5. Will R, Edmunds L, Elswood J, Calin A. Is There Sexual Inequality in Ankylosing Spondylitis? A Study of 498 Women and 1202 Men. J Rheumatol (1990) 17:1649-52.

6. Lee W, Reveille JD, Davis JCJr., Learch TJ, Ward MM, Weisman MH. Are There Gender Differences in Severity of Ankylosing Spondylitis? Results From the PSOAS Cohort. Ann Rheum Dis (2007) 66:633-8. doi: 10.1136/ ard.2006.060293

7. Schlosstein L, Terasaki PI, Bluestone R, Pearson CM. High Association of an HL-a Antigen, W27, With Ankylosing Spondylitis. N Engl J Med (1973) 288:704-6. doi: 10.1056/nejm197304052881403

8. Mear JP, Schreiber KL, Münz C, Zhu X, Stevanović S, Rammensee HG, et al. Misfolding of HLA-B27 as a Result of its B Pocket Suggests a Novel Mechanism for its Role in Susceptibility to Spondyloarthropathies. J Immunol (1999) 163:6665-70.

9. Goodall JC, Wu C, Zhang Y, McNeill L, Ellis L, Saudek V, et al. Endoplasmic Reticulum Stress-Induced Transcription Factor, CHOP, Is Crucial for Dendritic Cell IL-23 Expression. Proc Natl Acad Sci USA (2010) 107:17698-703. doi: 10.1073/pnas.1011736107

10. Bartels LE, Pedersen AB, Kristensen NR, Vilstrup H, Stengaard-Pedersen K, Dahlerup JF. A Positive Helicobacter Pylori Test Is Associated With Low Spondylarthritis Incidence in a Danish Historical Cohort Study. Rheumatol Int (2020) 40:359-66. doi: 10.1007/s00296-019-04487-2

11. Wei JC, Chou MC, Huang JY, Chang R, Hung YM. The Association Between Candida Infection and Ankylosing Spondylitis: A Population-Based Matched Cohort Study. Curr Med Res Opin (2020) 36:2063-9. doi: 10.1080/ 03007995.2020.1838460

12. Fragoulis GE, Liava C, Daoussis D, Akriviadis E, Garyfallos A, Dimitroulas T. Inflammatory Bowel Diseases and Spondyloarthropathies: From Pathogenesis to Treatment. World J Gastroenterol (2019) 25:2162-76. doi: 10.3748/ wjg.v25.i18.2162

13. Asquith M, Rosenbaum JT. The Interaction Between Host Genetics and the Microbiome in the Pathogenesis of Spondyloarthropathies. Curr Opin Rheumatol (2016) 28:405-12. doi: 10.1097/bor.0000000000000299

14. Rizzo A, Ferrante A, Guggino G, Ciccia F. Gut Inflammation in Spondyloarthritis. Best Pract Res Clin Rheumatol (2017) 31:863-76. doi: 10.1016/j.berh.2018.08.012

15. Zhang L, Hu Y, Xu Y, Li P, Ma H, Li X, et al. The Correlation Between Intestinal Dysbiosis and the Development of Ankylosing Spondylitis. Microb Pathog (2019) 132:188-92. doi: 10.1016/j.micpath.2019.04.038

16. Smith JA. Update on Ankylosing Spondylitis: Current Concepts in Pathogenesis. Curr Allergy Asthma Rep (2015) 15:489. doi: 10.1007/s11882-014-0489-6

17. Nielsen PR, Kragstrup TW, Deleuran BW, Benros ME. Infections as Risk Factor for Autoimmune Diseases - A Nationwide Study. J Autoimmun (2016) 74:176-81. doi: 10.1016/j.jaut.2016.05.013

18. Stone MA, Payne U, Schentag C, Rahman P, Pacheco-Tena C, Inman RD. Comparative Immune Responses to Candidate Arthritogenic Bacteria do Not Confirm a Dominant Role for Klebsiella Pneumonia in the Pathogenesis of Familial Ankylosing Spondylitis. Rheumatol (Oxford) (2004) 43:148-55. doi: 10.1093/rheumatology/keg482

19. Wei CY, Lin JY, Wang YT, Huang JY, Wei JC, Chiou JY. Risk of Ankylosing Spondylitis Following Human Papillomavirus Infection: A Nationwide, Population-Based, Cohort Study. J Autoimmun (2020) 113:102482. doi: 10.1016/j.jaut.2020.102482

20. Damba JJ, Laskine M, Jin Y, Sinyavskaya L, Durand M. Incidence of Autoimmune Diseases in People Living With HIV Compared to a Matched Population: A Cohort Study. Clin Rheumatol (2020) 40:2439-45. doi: 10.1007/ s10067-020-05500-X
21. Ratz T, Dean LE, Atzeni F, Reeks C, Macfarlane GJ, Macfarlane TV. A Possible Link Between Ankylosing Spondylitis and Periodontitis: A Systematic Review and Meta-Analysis. Rheumatol (Oxford) (2015) 54:500-10. doi: 10.1093/rheumatology/keu356

22. Keller JJ, Kang JH, Lin HC. Association Between Ankylosing Spondylitis and Chronic Periodontitis: A Population-Based Study. Arthritis Rheum (2013) 65:167-73. doi: 10.1002/art.37746

23. Yen YF, Chuang PH, Jen IA, Chen M, Lan YC, Liu YL, et al. Incidence of Autoimmune Diseases in a Nationwide HIV/AIDS Patient Cohort in Taiwan, 2000-2012. Ann Rheum Dis (2017) 76:661-5. doi: 10.1136/annrheumdis2016-209815

24. Chu KA, Chen W, Hung YM, Wei JC. Increased Risk of Ankylosing Spondylitis After Mycoplasma Pneumonia: A Nationwide Population-Based Study. Med (Baltimore) (2019) 98:e15596. doi: 10.1097/md.0000000000015596

25. Feng XG, Xu XJ, Ye S, Lin YY, Chen P, Zhang XJ, et al. Recent Chlamydia Pneumoniae Infection Is Highly Associated With Active Ankylosing Spondylitis in a Chinese Cohort. Scand J Rheumatol (2011) 40:289-91. doi: 10.3109/03009742.2011.560891

26. Lindström U, Exarchou S, Lie E, Dehlin M, Forsblad-d'Elia H, Askling J, et al. Childhood Hospitalisation With Infections and Later Development of Ankylosing Spondylitis: A National Case-Control Study. Arthritis Res Ther (2016) 18:240. doi: 10.1186/s13075-016-1141-8

27. Chao WC, Lin CH, Chen YM, Jiang RS, Chen HH. Association Between Tonsillitis and Newly Diagnosed Ankylosing Spondylitis: A Nationwide, Population-Based, Case-Control Study. PloS One (2019) 14:e0220721. doi: 10.1371/journal.pone.0220721

28. Abbood HM, Pathan E, Cherukara GP. The Link Between Ankylosing Spondylitis and Oral Health Conditions: Two Nested Case-Control Studies Using Data of the UK Biobank. J Appl Oral Sci (2018) 27:e20180207. doi: 10.1590/1678-7757-2018-0207

29. Stroup DF, Berlin JA, Morton SC, Olkin I, Williamson GD, Rennie D, et al. Meta-Analysis of Observational Studies in Epidemiology: A Proposal for Reporting. Meta-Analysis of Observational Studies in Epidemiology (MOOSE) Group. Jama (2000) 283:2008-12. doi: 10.1001/jama.283.15.2008

30. Page MJ, Moher D. Evaluations of the Uptake and Impact of the Preferred Reporting Items for Systematic Reviews and Meta-Analyses (PRISMA) Statement and Extensions: A Scoping Review. Syst Rev (2017) 6:263. doi: 10.1186/s13643-017-0663-8

31. Xuan K, Jha AR, Zhao T, Uy JP, Sun C. Is Periodontal Disease Associated With Increased Risk of Colorectal Cancer? A Meta-Analysis. Int J Dent Hyg (2021) 19:50-61. doi: 10.1111/idh.12483

32. Wells GA, Shea B, O'Connell D, Peterson J, Welch V, Losos M, et al. The Newcastle-Ottawa Scale (NOS) for Assessing the Quality of Nonrandomised Studies in Meta-Analyses (2021). Available at: http://www.ohri.ca/programs/ clinical_epidemiology/oxford.asp (Accessed September 26, 2021).

33. Higgins JPT, Thomas J, Chandler J, Cumpston M, Li T, Page MJ, et al. Cochrane Handbook for Systematic Reviews of Interventions Version 6.2 (2021). Cochrane. Available at: http://www.training.cochrane.org/handbook (Accessed October 6, 2021). updated February 2021.

34. Huai P, Xun H, Reilly KH, Wang Y, Ma W, Xi B. Physical Activity and Risk of Hypertension: A Meta-Analysis of Prospective Cohort Studies. Hypertension (2013) 62:1021-6. doi: 10.1161/HYPERTENSIONAHA.113.01965

35. Bartels LE, Jepsen P, Christensen LA, Gerdes LU, Vilstrup H, Dahlerup JF. Diagnosis of Helicobacter Pylori Infection Is Associated With Lower Prevalence and Subsequent Incidence of Crohn's Disease. J Crohns Colitis (2016) 10:443-8. doi: 10.1093/ecco-jcc/jjv229

36. Yap TW, Gan HM, Lee YP, Leow AH, Azmi AN, Francois F, et al. Helicobacter Pylori Eradication Causes Perturbation of the Human Gut Microbiome in Young Adults. PloS One (2016) 11:e0151893. doi: 10.1371/journal.pone.0151893

37. Yang L, Wang L, Wang X, Xian CJ, Lu H. A Possible Role of Intestinal Microbiota in the Pathogenesis of Ankylosing Spondylitis. Int J Mol Sci (2016) 17:2126. doi: 10.3390/ijms17122126

38. Al-Katma MK, Bissada NF, Bordeaux JM, Sue J, Askari AD. Control of Periodontal Infection Reduces the Severity of Active Rheumatoid Arthritis. J Clin Rheumatol (2007) 13:134-7. doi: 10.1097/RHU.0b013e3180690616

39. de Pablo P, Dietrich T, McAlindon TE. Association of Periodontal Disease and Tooth Loss With Rheumatoid Arthritis in the US Population. J Rheumatol (2008) 35:70-6 
40. Pischon N, Pischon T, Kröger J, Gülmez E, Kleber BM, Bernimoulin JP, et al. Association Among Rheumatoid Arthritis, Oral Hygiene, and Periodontitis. J Periodontol (2008) 79:979-86. doi: 10.1902/jop.2008.070501

41. Palomares O, Rückert B, Jartti T, Kücüksezer UC, Puhakka T, Gomez E, et al. Induction and Maintenance of Allergen-Specific FOXP3+ Treg Cells in Human Tonsils as Potential First-Line Organs of Oral Tolerance. J Allergy Clin Immunol (2012) 129:510-20, 20.e1-9. doi: 10.1016/j.jaci. 2011.09.031

42. Itescu S, Dalton J, Zhang HZ, Winchester R. Tissue Infiltration in a CD8 Lymphocytosis Syndrome Associated With Human Immunodeficiency Virus1 Infection has the Phenotypic Appearance of an Antigenically Driven Response. J Clin Invest (1993) 91:2216-25. doi: 10.1172/jci116448

43. Fife DJ, Waller JM, Jeffes EW, Koo JY. Unraveling the Paradoxes of HIVAssociated Psoriasis: A Review of T-Cell Subsets and Cytokine Profiles. Dermatol Online J (2007) 13:4. doi: 10.5070/D34SF63339

44. Russo S, Lopalco L. Is Autoimmunity a Component of Natural Immunity to HIV? Curr HIV Res (2006) 4:177-90. doi: 10.2174/157016206776055011
Conflict of Interest: The authors declare that the research was conducted in the absence of any commercial or financial relationships that could be construed as a potential conflict of interest.

Publisher's Note: All claims expressed in this article are solely those of the authors and do not necessarily represent those of their affiliated organizations, or those of the publisher, the editors and the reviewers. Any product that may be evaluated in this article, or claim that may be made by its manufacturer, is not guaranteed or endorsed by the publisher.

Copyright (c) 2021 Zhang, Sun, Zhou, Tao, Chen, Shi, Yin, Sun and Ding. This is an open-access article distributed under the terms of the Creative Commons Attribution License (CC BY). The use, distribution or reproduction in other forums is permitted, provided the original author(s) and the copyright owner(s) are credited and that the original publication in this journal is cited, in accordance with accepted academic practice. No use, distribution or reproduction is permitted which does not comply with these terms. 